\title{
Cepheid Radial Velocities and Phase Lag
}

\author{
M. E. Sachkov \\ Institute of Astronomy, 48 Pyatnitskaya Str., Moscow 109017, Russia
}

\begin{abstract}
Our systematic measurements (since 1987) of radial velocities of northern Cepheids with a correlation spectrometer allowed us to make a detailed study of shapes of $V_{\mathrm{R}}$ curves. This is a preliminary analysis of the phase lag between Cepheid radial velocity and light curves.
\end{abstract}

As was believed until recently, the hump (secondary minimum) in the $V_{\mathbf{R}}$ curve is positioned at the same phase as the hump (secondary maximum) on the light curve (Fadeev 1994). But detailed analysis of $V_{R}$ curves shows that, in most cases, light and radial velocity curves shapes are significantly different (Sachkov et al. 1998).

To study the phase lag (in fractions of the pulsational period) between humps of light ( $V$ magnitude) and $V_{\mathrm{R}}$ curves, we used our radial velocity measurements (Gorynya et al. 1992, 1996b, 1998) and photoelectric observations (Berdnikov 1995). This investigation requires simultaneous sets of spectroscopic and photometric observations. We have considered sets simultaneous if they cross in time and have a duration of several pulsational periods. We used the so-called standard curves which fix the shape of the curve for each star (Sachkov et al. 1998).

For analysis, we selected 36 Cepheids. Our sample contains only DCEP variables according to the classification system of the GCVS, which presumably pulsate in the fundamental mode. For spectroscopic binaries we used pulsational radial velocity curves (after taking into account orbital motion, with elements from Gorynya, Rastorgouev, \& Samus (1996a)).

The correlation of the difference between radial velocity and light humps phases upon logarithm of period can be represented (see also Fig. 1) as:

phase(hump $\left.V_{\mathrm{R}}\right)$-phase (hump $\left.V\right)=0.15( \pm 0.03) \log P+0.02( \pm 0.02)$.

We also estimated the difference between phase of maximum of radial velocity and phase of minimum of light. This difference probably has no dependence on period and can be considered constant, equal to $0.06 \pm 0.03$ (in fractions of the period).

Acknowledgments. The author is grateful to Drs. N. A. Gorynya, N. N. Samus, and A. S. Rastorgouev for helpful discussions, and Mrs. Sachkova for assistance. Thanks are due to the Council of the Program of Support for Scientific Schools (grant 96-15-96656). 


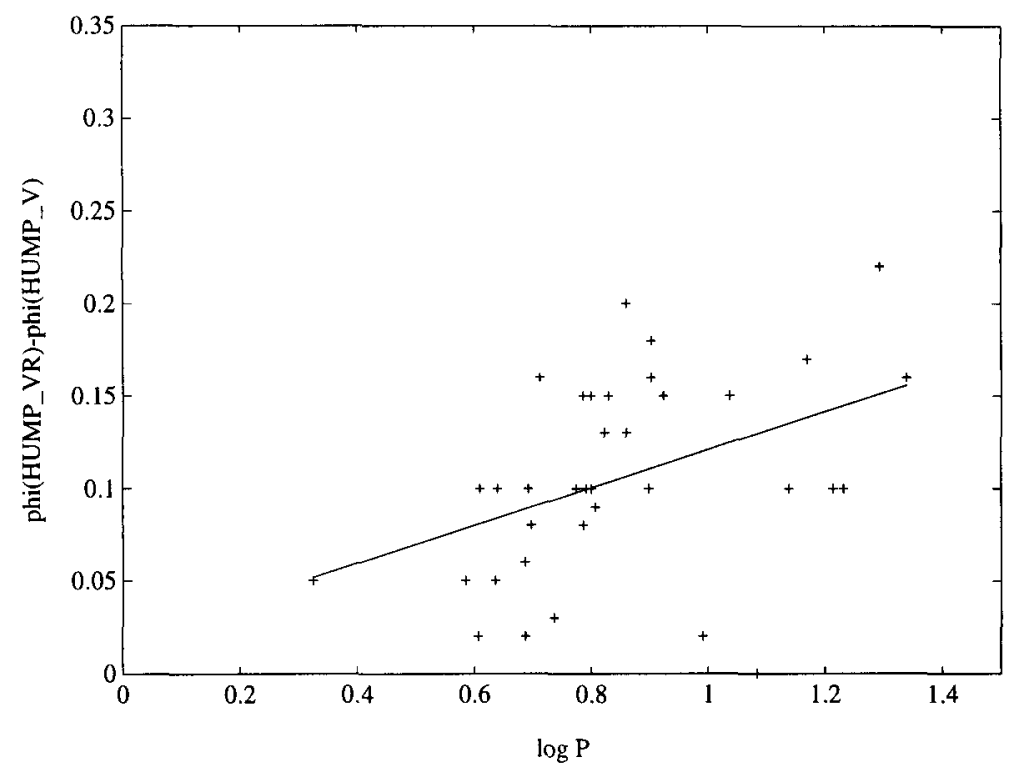

Figure 1. The correlation of the difference between radial velocity and photometric hump phases vs. logarithm of pulsational period.

\section{References}

Berdnikov, L. N. 1995, in ASP Conf. Ser. Vol. 83, Astrophysical Applications of Stellar Pulsation, ed. R. S. Stobie \& P. A. Whitelock (San Francisco: ASP), 207

Fadeev, Yu. A. 1994, in Unstable Processes in the Universe, ed. A. G. Masevich, (Moscow: Kosmosinform), 79 (in Russian)

Gorynya, N. A., Irsmambetova, T. R., Rastorgouev, A. S., \& Samus, N. N. 1992, Astr. Letters, 18, 316

Gorynya, N. A., Rastorgouev, A. S., \& Samus, N. N. 1996a, Astr. Letters, 22, 33

Gorynya, N. A., Samus, N. N., Rastorgouev, A. S., \& Sachkov, M. E. 1996b, Astr. Letters, 22, 175

Gorynya, N. A., Samus, N. N., Sachkov, M. E., et al. 1998, Astr. Letters, 24, 815

Sachkov, M. E., Rastorgouev, A. S., Samus, N. N., \& Gorynya, N. A. 1998, Astr. Letters, 24, 377 